\title{
Docking and electron transfer studies between rubredoxin and rubredoxin : oxygen oxidoreductase
}

\section{Serviços $9^{\circ}$ on \\ $\mathrm{NCBI}$}

\author{
Print E-mail Add to Marked List Save to EndNote Web \\ Save to EndNote, RefMan, ProCite more options
}

Author(s): Victor BL, Vicente JB, Rodrigues R, Oliveira S, Rodrigues-Pousada C, Frazao C, Gomes CM, Teixeira M, Soares CM

Source: JOURNAL OF BIOLOGICAL INORGANIC CHEMISTRY Volume: 8 Issue: 4 Pages: 475488 Published: APR 2003

\section{Times Cited: 9 References: $81 \quad$ Ex ECitation Map}

\begin{abstract}
The interaction and electron transfer (ET) between rubredoxin (Rd) and rubredoxin: oxygen oxidoreductase (ROO) from Desulfovibrio gigas is studied by molecular modelling techniques. Experimental kinetic assays using recombinant proteins show that the Rd reoxidation by $\mathrm{ROO}$ displays a bell-shaped dependence on ionic strength, suggesting a non-trivial electrostatic dependence of the interaction between these two proteins. Rigid docking studies reveal a prevalence for Rd to interact, in a very specific way, with the surface of the ROO dimer near its FMN cofactors. The optimization of the lowest energy complexes, using molecular dynamics simulation, shows a very tight interaction between the surface of the two proteins, with a high probability for Rd residues (but not the iron centre directly) to be in direct contact with the FMN cofactors of ROO. Both electrostatics and van der Waals interactions contribute to the final energy of the complex. In these complexes, the major contributions for complex formation are polar interactions between acidic residues of Rd and basic residues of ROO, plus substantial non-polar interactions between different groups. Important residues for this process are identified. ET estimates (using the Pathways model), in the optimized lowest energy complexes, suggest that these configurations are efficient for transferring electrons. The experimental bell-shaped dependence of kinetics on ionic strength is analysed in view of the molecular modelling results, and hypotheses for the molecular basis of this phenomenon are discussed.
\end{abstract}

Document Type: Article

Language: English

Author Keywords: protein-protein interactions; molecular dynamics refinement; electron transfer; oxygen and NO metabolism; ionic strength dependence

KeyWords Plus: CYTOCHROME-C PEROXIDASE; ANAEROBE DESULFOVIBRIO-GIGAS; PROTEINPROTEIN INTERACTIONS; MOLECULAR-DYNAMICS; SOFT-DOCKING; ESCHERICHIA-COLI; SHAPE COMPLEMENTARITY; TRANSFER COMPLEXES; REDUCING BACTERIA; AUTOMATED DOCKING

Reprint Address: Soares, CM (reprint author), Univ Nova Lisboa, Inst Tecnol Quim \& Biol, Apt 127,Av Republ, P-2781901 Oeiras, Portugal

Addresses:

1. Univ Nova Lisboa, Inst Tecnol Quim \& Biol, P-2781901 Oeiras, Portugal

2. Univ Evora, Dept Biol, P-7000 Evora, Portugal

3. Univ Nova Lisboa, Fac Ciencias \& Tecnol, Dept Quim, P-2825114 Caparica, Portugal

Publisher: SPRINGER-VERLAG, 175 FIFTH AVE, NEW YORK, NY 10010 USA 